\title{
Optomechanical self-structuring in cold atomic gases
}

\author{
G. Labeyrie ${ }^{1 *}$, E. Tesio ${ }^{2}$, P. M. Gomes ${ }^{2}$, G.-L. Oppo ${ }^{2}$, W. J. Firth ${ }^{2}$, \\ G. R. M. Robb ${ }^{2}$, A. S. Arnold ${ }^{2}$, R. Kaiser ${ }^{1,2 *}$, and T. Ackemann ${ }^{2 *}$ \\ ${ }^{1}$ Institut Non Linéaire de Nice, UMR 7335 CNRS, \\ 1361 route des Lucioles, 06560 Valbonne, France and \\ ${ }^{2}$ SUPA and Department of Physics, University of Strathclyde,Glasgow G4 0NG, Scotland, UK \\ (Dated: Author's last version, published as Nature Phot. 8, 321325 (2014).) \\ (Www.nature.com/nphoton/journal/v8/n4/abs/nphoton.2014.52.html)
}

The rapidly developing field of optomechanics aims at the combined control of optical and mechanical modes 1 . In cold atoms, the spontaneous emergence of spatial structures due to optomechanical back-action has been observed in one dimension, in optical cavities ${ }^{3}$ or highly anisotropic samples ${ }^{8}$. Extensions to higher dimensions aiming to exploit multi-mode configurations have been suggested theoretically ${ }^{15}$. We describe here a simple experiment with many spatial degrees of freedom, in which two continuous symmetries - rotation and translation in the plane orthogonal to a pump beam axis - are spontaneously broken. We observe the simultaneous long range spatial structuring, with hexagonal symmetry, of the density of a cold atomic cloud and of the pump optical field, with adjustable length scale. Being based on coherent phenomena (diffraction and the dipole force), this scheme can potentially be extended to quantum degenerate gases.

In mutually coupled light-atom systems, a pump field interacts with a cloud of cold atoms and a probe field (possibly a noise field) propagating along the same or a separate axis 69 . The interference pattern created by these two fields causes a bunching of the cold atoms due to dipole forces. The resulting density grating then scatters pump light into the probe mode. Examples involving the generation of new coherent waves from optomechanical back-action are recoil induced resonances ${ }^{16}$, superradiant Rayleigh scattering ${ }^{8}$, collective atomic recoil lasing (CARL $)^{6}$ and self-organization in transversely pumped spatially single-mode cavities 57 . The probe field is thus amplified and it further enhances the density grating. Starting from noise, a coherent field output is obtained for the probe mode and this transition to a modulated light and density pattern can be understood as a bifurcation or a dynamical phase transition 5 .

Further theoretical work addresses the possibility of multi-mode structures in transversely pumped cavities 10111 and in systems in which the pump axis is

\footnotetext{
* To whom correspondence should be addressed.
}

the only distinguished axis 912 . In the latter, twodimensional purely optomechanical structures emerge spontaneously in the plane transverse to the pump axis. The length scale selection via diffraction leads to a high number of degenerate modes on a circle in transverse Fourier space, as in the Brazowskii phase transition from isotropic to nonuniform states in Hamiltonian systems 17 . Here we report such structures in cold atoms, augmenting the realizations of the beautiful phenomenon of spontaneous self-organization in systems driven out of thermal equilibrium with broad relevance to natural sciences and technology by atomic density structures. It allows for the remote, contact-free creation and manipulation of complex matter distributions to be sustained by homogenous driving only.

While self-organized diffractive structures have been observed in hot atomic vapours for a number of years 18 , these rely solely on the spatial modulation of the internal states of the atoms (electronic or Zeeman states) without affecting their centre-of-mass motion. The crucial new ingredient in cold atoms is the existence of macroscopic matter transport processes due to dipole forces, leading to density self-organization without the need for an intrinsic optical nonlinearity (first experimental clues of such an effect in a very low aspect ratio situation were obtained in ${ }^{9}$ ). Compared to soft matter systems 2123 cold atoms have the advantage that the dynamics can be studied without viscous damping of motion, allowing for a dissipation free evolution with a Hamiltonian description of the system. It is possible, however, to introduce dissipation in a controlled way, if desired, via optical molasses $6[9 / 13 / 14$. From the photonics and quantum optics point of view, cold atomic systems enable the prospect of very low thresholds 914415 with the long-term vision to achieve all-optical control of symmetry breaking at the single photon leve $120 \mid 24$.

An effective and conceptually simple scheme for optical pattern formation is the single mirror feedback arrangement ${ }^{25 / 26}$ illustrated in Fig. 1 1 a. It is based on a pump laser beam passing through an optical nonlinear medium (NL, here a cloud of laser-cooled atoms) and being retro-reflected by a plane mirror $(\mathrm{M})$ located at a distance $d$. Any spatial perturbation of the transmitted wavefront due to small fluctuations of the cloud refractive index $n$ along the transverse directions can convert into 

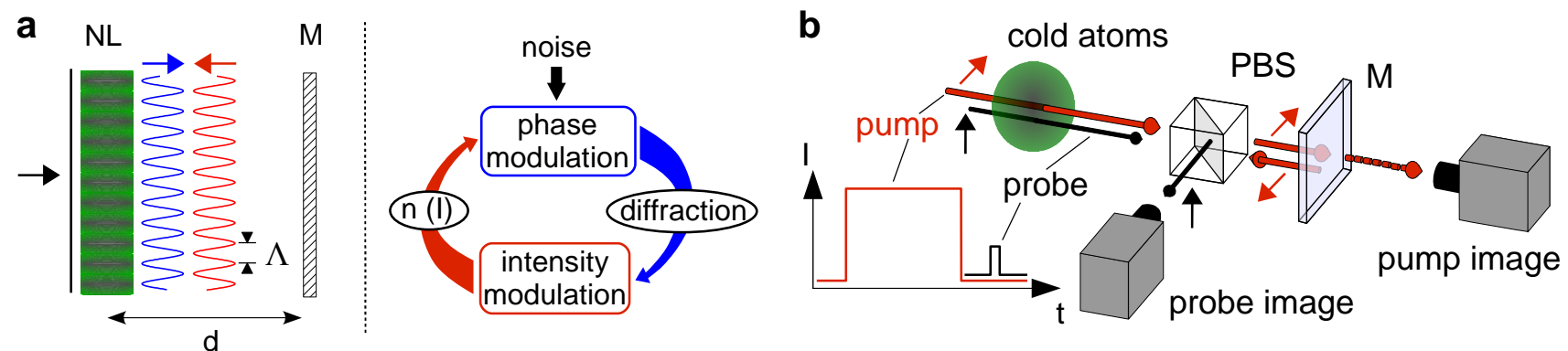

FIG. 1: Self-organization scheme and experimental setup. a, Mechanism for self-organization in the single mirror feedback scheme. b, Experimental setup and timing diagram for pump and probe pulses (NL: nonlinear medium, PBS: polarizing beam splitter, M: mirror).

an intensity perturbation because of diffraction during the free-space propagation to the mirror and back to the cloud. Since the refractive index of the cloud depends on the light intensity $I$, these intensity perturbations further modify the transverse distribution of the refractive index and produce a feedback loop. If this feedback is positive, its effect is to enhance the initial index fluctuations leading to the growth of a macroscopic transverse modulation of both light intensity and refractive index, and to the spontaneous appearance of patterns. The typical length scale $\Lambda$ of the emerging pattern is set by diffraction and can be adjusted by varying the feedback distance $25+27$.

The index of refraction of our cold atomic cloud can be written in the form

$$
n(I, \nabla I)=1-\frac{3 \lambda^{3}}{4 \pi^{2}} \frac{\delta / \Gamma}{1+(2 \delta / \Gamma)^{2}} \frac{\rho(\nabla I)}{1+s(I)}
$$

where $\delta=\omega_{1}-\omega_{0}$ is the detuning between the laser and atomic frequencies, $\Gamma$ is the natural width, $\nabla I$ the gradient of the intensity and $\rho$ denotes the atomic density. The saturation parameter is $s=I /\left[I_{\text {sat }}\left(1+4(\delta / \Gamma)^{2}\right)\right]$, where $I_{\text {sat }}$ is the saturation intensity. This expression exhibits two distinct mechanisms which can lead to positive feedback. One is due to the $1 /(1+s)$ term in Eq. (1), converting spatial intensity modulations into refractive index modulations via changes of the population of the excited state (an internal degree of freedom). This is the well-known 'two-level' nonlinearity. It is negligible for $s \ll 1$ (linear regime) and produces a Kerr-like nonlinear refractive index $\left(n \simeq n_{0}+n_{2} I\right)$ for intermediate saturation $(s<1)$. The two-level nonlinearity is selffocusing ( $n$ increases with $I$ ) for $\delta>0$.

In this work, we focus on the second mechanism, an optomechanical nonlinearity which arises from the $\rho(\nabla I)$ term in Eq. (1). The coupling between $\nabla I$ and atomic density is due to the dipole force exerted on the cold atoms in the presence of transverse intensity variations, which results in spatial bunching. For $\delta>0$ the atoms are expelled from high-intensity areas, $\rho$ decreases and hence $n$ increases where the intensity $I$ is high, leading to an effectively self-focusing nonlinearity, though the mechanism is nonlocal in principle. This effect typically becomes important when the depth of the dipole potential is similar to or exceeds the kinetic energy of the atoms (set by the cold cloud temperature). We stress that the optomechanical coupling can in principle sustain an instability even in the linear optical regime $(s \ll 1)$, provided that the temperature of the atomic ensemble is low enough $^{28}$ (see also the end of Sect. II of the Supplementary Material).

In our experiment sketched in Fig. $1 \mathrm{~b},{ }^{87} \mathrm{Rb}$ atoms are released from a large magneto-optical trap (MOT) at a temperature of $290 \mu \mathrm{K}$. The collimated and linearlypolarized pump beam is retro-reflected by the mirror after passing through the cloud. An orthogonallypolarized, weak probe beam is sent along the same optical path through the cloud, after the interaction with the pump, to probe subsequent spatial ordering. Typical images of the transmitted pump beam intensity profile exhibit a high-contrast hexagonal pattern (Fig. 2a, left), which extends over a large portion of the beam crosssection. The intensity in the bright spots of this pattern is more than three times larger than the peak intensity of the incident pump beam. We typically observe patterns for $3 \Gamma<\delta<25 \Gamma, I_{\text {in }}>50 \mathrm{~mW} / \mathrm{cm}^{2}$, and for a cloud optical density $(O D)$ above a threshold value which depends on the pump pulse duration (see Sect. I.B of the Supplementary Material). The pattern in the pump beam is accompanied by a spatial self-structuring of the atomic cloud, as measured by the probe beam (Fig. 2a, right). This atomic density image (see details in 'Methods') displays a high-contrast honeycomb pattern complementary to the hexagonal pattern of the pump, as expected. A further confirmation of this spatial bunching of the atoms is obtained by measuring the decay of the probe pattern as the time delay between pump and probe pulses is increased (Fig. 3a). We observe a slow decay (time scale $\approx 80 \mu \mathrm{s}$ ) excluding the possibility of a transverse spatial modulation of the excited state population (which has a time constant of $\Gamma^{-1}=26 \mathrm{~ns}$ ), but is compatible with 

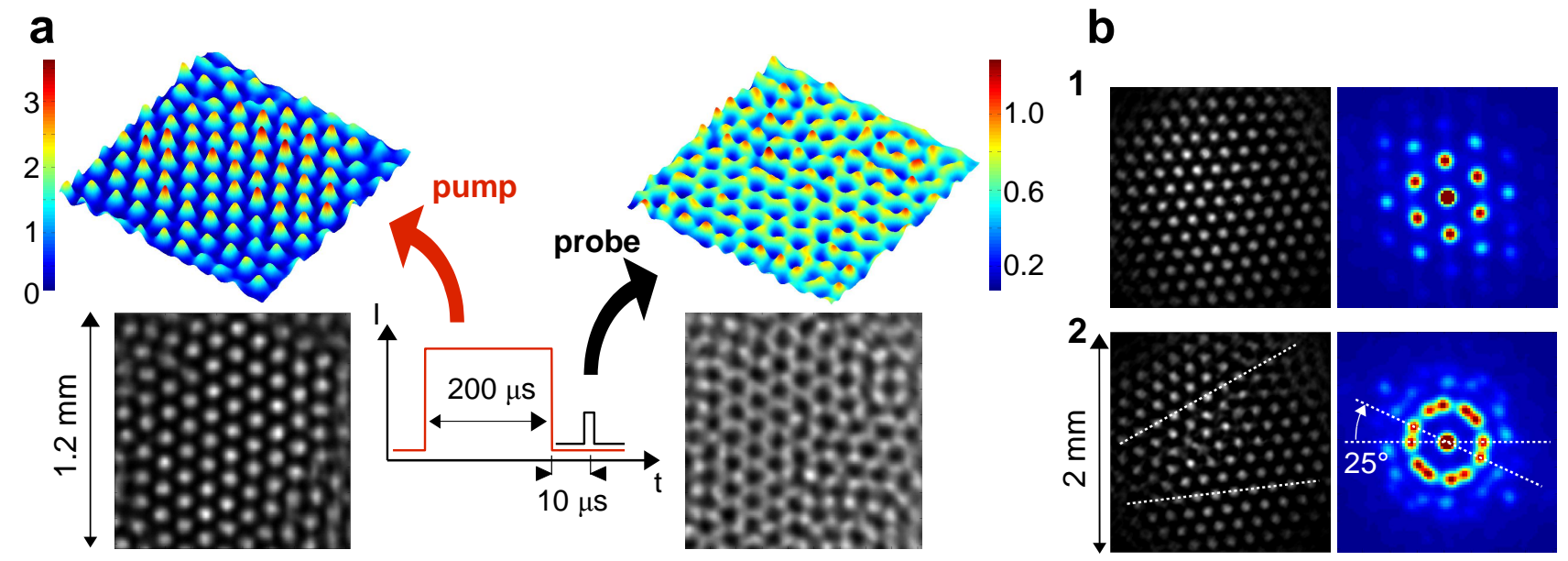

FIG. 2: Observation of self-organization. a, Transmission images (normalized to the intensities of the transmitted beam recorded in the absence of atoms) recorded with pump and probe (3D intensity distributions shown in colour). Parameters are: input intensity $I_{\mathrm{in}}=129 \mathrm{~mW} / \mathrm{cm}^{2}, \delta=+7 \Gamma$, and $d=5 \mathrm{~mm}$. b. Continuous symmetry breaking and defect formation. 1 shows an extended pattern in the transmitted beam with long-range order (the colour image is a numerically calculated Fourier transform of the intensity pattern showing hexagonal symmetry). In 2, displaying another realization of the same experiment as $\mathbf{1}$, the hexagonal structures in the upper left and lower right have different orientations leading to a defect line.

ballistic dephasing of the atomic density structures once the pump pulse has ended. Instability mechanisms based on the spatial modulation of Zeeman or hyperfine states were excluded by further measurements (see Sect. I.A of the Supplementary Material).

The observation of a strong transverse spatial bunching is consistent with the fact that the estimated dipole potential height lies in the hundreds of $\mu \mathrm{K}$ to $\mathrm{mK}$ range, comparable to or larger than the MOT temperature. (We discuss the possibility of a longitudinal bunching on wavelength scales in Sect. I.C of the Supplementary Material). It is also in qualitative agreement with predictions from a model describing the coupled dynamics of the light field and the atomic density (see Sect. II of the Supplementary Material). This observation thus constitutes direct evidence of spontaneous transverse organization and symmetry breaking of the atomic density under the action of a single retro-reflected laser beam. Fig. $2 \mathrm{~b}$ illustrates continuous symmetry breaking and the occurrence of defects. In most cases, a long-range ordered pattern is observed (1) with small and random shot-to-shot variations of both position and orientation. Occasionally however, a topological defect is observed $(\mathbf{2})$ with a boundary separating regions which chose different orientations in the symmetry breaking process.

The tunability of the pattern length scale $\Lambda$ is illustrated in Fig. 3b. The dependence of the length scale on the mirror distance confirms that the conversion from phase to intensity fluctuations in the feedback loop is the key ingredient for the occurrence of the instability. The experimental results (circles) are in excellent agreement with the prediction from a thick-medium model (squares, see Sect. I.C of the Supplementary Material).

Simultaneous monitoring of light patterns on the pump and the probe provides insights into the instability mechanisms at work. We measured the evolution of the pattern contrast in the pump and probe as the duration and intensity of the pump pulse was varied (Fig. 4). The observed increase of contrast with pump duration, on a time scale (several tens of $\mu \mathrm{s}$ ) much larger than the excited state decay time is a clear manifestation of the optomechanical nonlinearity. This interpretation is supported by the comparison of the pump and probe patterns at the shortest pump duration $(1 \mu \mathrm{s})$ : while the probe pattern is always absent, the pump pattern is on the contrary already sizable for large enough intensities (see curves (1) and (2) in Fig. 4). This is interpreted to be a regime in which the two-level nonlinearity alone can trigger pattern formation, i.e. curves (1) and (2) are above the two-level instability threshold. Afterwards, the rather 'slow' optomechanical nonlinearity leads to atomic bunching, resulting in turn in a significant enhancement of the contrast of the light pattern. For low pump intensity (curve (4) in Fig. 4), one observes a co-evolution of both pump and probe patterns, with patterns appearing in the atomic density and light intensity only after a relatively long pump duration. In this regime, the system is below the two-level instability threshold and the selforganization is clearly dominated by the optomechanical nonlinearity. For the parameters of curve (4), the saturation parameter corresponding to the incident pump is only 0.06. Pattern formation at even smaller saturation values should be possible (see Fig. S2 of the Supplementary Material), provided that a colder atomic cloud is 

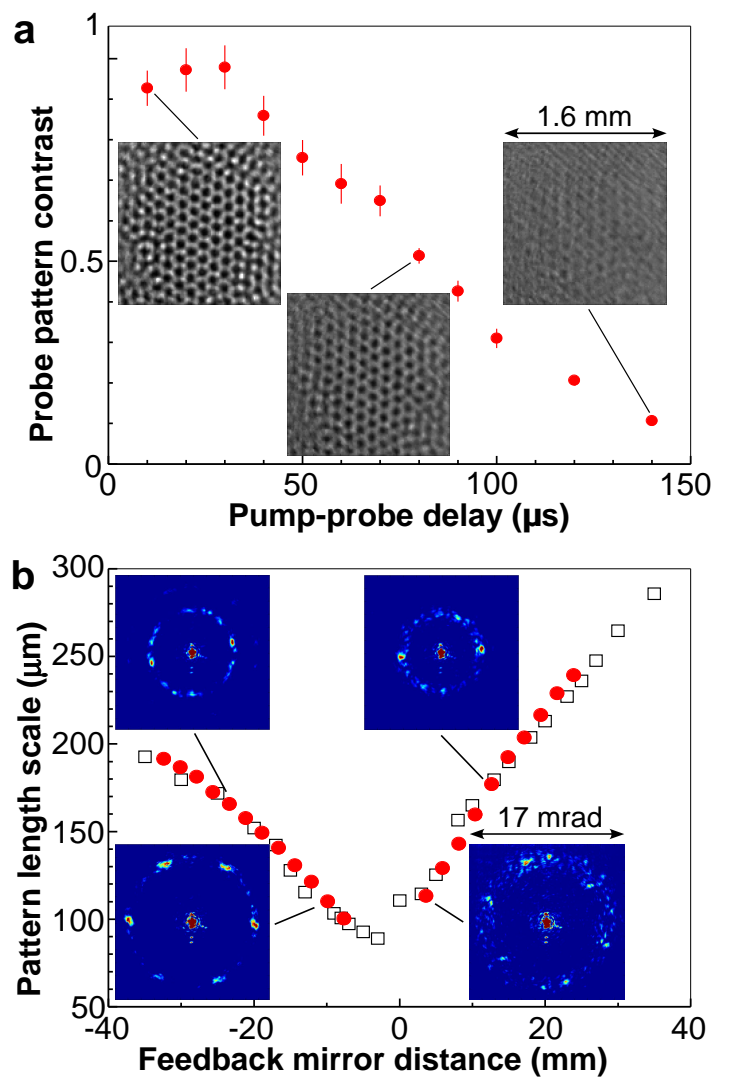

FIG. 3: Pattern temporal lifetime and spatial period. a, Decay of density pattern with increasing pump-probe delay. The long decay constant is attributed to atomic motion. b, Pattern length scale vs feedback mirror distance. Measured sizes (circles) are obtained from far-field transmission images (insets); squares are from a thick-medium model (see Sect. I.C of the Supplementary Material). The error bars correspond to four times the standard deviation obtained from 10 realizations.

used, bringing the system even further into the optomechanical instability regime.

In conclusion, we have observed spontaneous transverse pattern formation in the transmitted light field and in the atomic density, with continuous symmetries breaking under the action of a single retro-reflected laser beam. Using a model based on optomechanical coupling with dipole forces for the atoms and diffraction of the light field, we find good agreement with the experimental results. This model allows us to conjecture that lowering the temperature of the atomic cloud will decrease the instability thresholds in terms of opacity of the atomic cloud as well as laser intensity, enabling low power control of both light and matter. As only coherent propagation of light and non-dissipative forces are required for the optomechanical self-structuring to occur, this scheme is expected to be viable with quantum degenerate ensembles of atoms. The continuous symmetry breaking asso- a
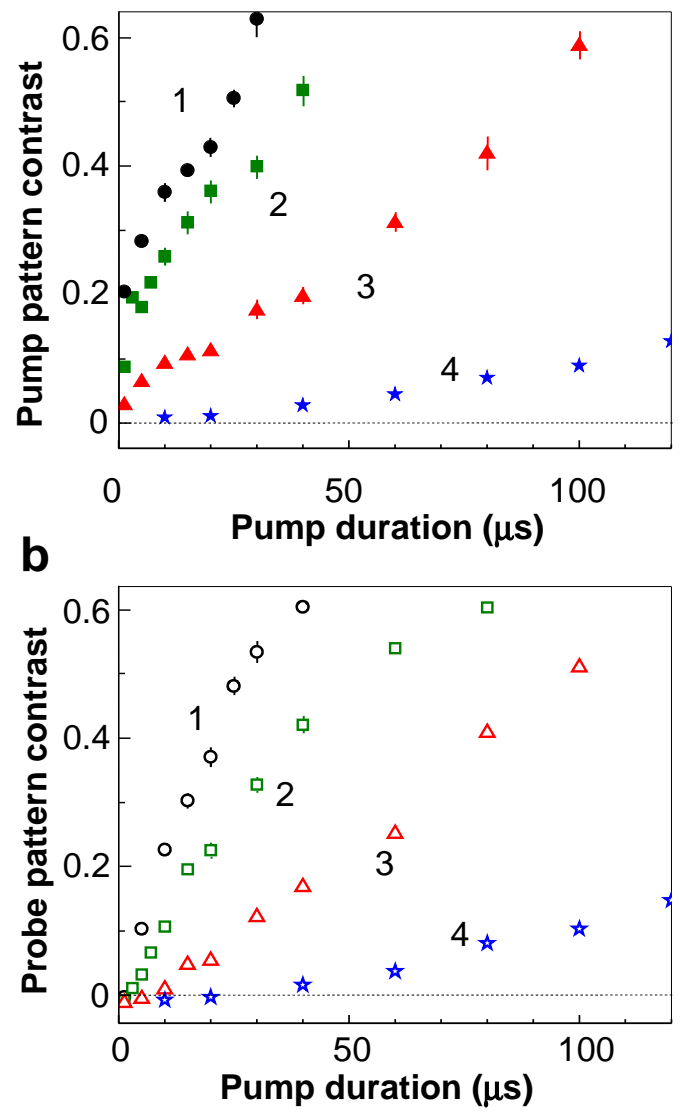

FIG. 4: Optomechanical vs two-level nonlinearity. Measured pattern contrast in pump (a) and probe (b) transmission images, as a function of pump pulse duration. Different pump intensities are used: $1, I_{\text {in }}=636 \mathrm{~mW} / \mathrm{cm}^{2} ; 2, I_{\text {in }}=$ $404 \mathrm{~mW} / \mathrm{cm}^{2} ; 3, I_{\text {in }}=217 \mathrm{~mW} / \mathrm{cm}^{2} ; 4, I_{\text {in }}=91 \mathrm{~mW} / \mathrm{cm}^{2}$. The error bars correspond to four times the standard deviation obtained from 20 realizations.

ciated with our scheme then potentially opens the way to study complex, multi-spatial mode situations $10[11$ including defect formation and dynamics. As the lattice is self-organized and not externally imposed, it is not rigid but can have dynamics leading to the possibility to study the propagation of coupled light-density perturbations, correlations of fluctuations in the light and density modes, and, in presence of atomic coherence, corresponding features in quantum transport.

The mechanism of self-structuring with optical feedback described here is expected to apply also to the electron density in a plasma in the presence of ponderomotive forces 28 , to 'soft matter' formed from dielectric beads22123, and deformable membranes 29 . Beside periodic patterns, one emerging feature suggested by theoretical simulations is the possibility of localized, soliton-like, density-light structures (single or multiple holes in the density), which can be set and erased by external control 
pulses and sustained by homogeneous driving only $\sqrt{30}$.

\section{Methods}

Sample preparation. The full experimental sequence alternates a preparation stage where the atoms are trapped and cooled, and a measurement stage where the trapping lasers and magnetic field of the MOT are switched off, the pump beam and then the probe beam are switched on and the transmission images are acquired. The atomic cloud has a Gaussian density profile with dimensions (full width at half maximum) of $(10 \times 10 \times 5) \mathrm{mm}^{3}(10 \mathrm{~mm}$ along the pump propagation axis), and contains $6 \times 10^{10}$ atoms. The peak spatial density is $10^{11} \mathrm{~cm}^{-3}$ corresponding to an $O D=-\ln \left(T_{I}\right)=$ 150 (where $T_{I}$ is the fraction of transmitted light intensity) at line centre (see alsd $\frac{31}{3}$ ).

Details of the experiment. The light-atom interaction described in this experiment is based on the $F=2 \rightarrow F^{\prime}=3$ transition of the $D_{2}$ line of ${ }^{87} \mathrm{Rb}$ at $780.24 \mathrm{~nm}$. The natural linewidth of this transition is $\Gamma=2 \pi \times 6.067 \mathrm{MHz}$ and the saturation intensity is $I_{\text {sat }}=3.58 \mathrm{~mW} / \mathrm{cm}^{2}$ assuming an equal population distribution among Zeeman substates. We stress that the distinction between the optomechanically- and two-level- triggered instabilities (see discussion of Fig. 4 is based on the experimental observation of the pattern formation dynamics, and does not rely on an estimate of the saturation level. All results presented here are obtained with a few percent of repumping light (compared to pump light) copropagating with the pump beam. It is tuned close to the $F=1 \rightarrow F^{\prime}=2$ transition. The pump beam is directed to the atoms via a monomode polarization-maintaining optical fibre. It is collimated and linearly-polarized (waist $1.6 \mathrm{~mm}$ ). The feedback mirror is located outside the vacuum chamber where the cold atoms are produced. We therefore use a telescope to image the mirror onto a plane located near the cold atomic cloud, producing a 'virtual mirror' whose distance to the sample can be adjusted from positive to negative values 27 . The typical saturation parameter range for the pump is between 0.05 and 1 . The image plane of the transmitted pump beam is located $\simeq 10 \mathrm{~mm}$ after the cloud centre. For the patterns shown in Figs. 2 and 3 a, this corresponds to the feedback beam reinjected into the medium.

Diffractive length scale selection. Within paraxial approximation, any periodic spatial modulation with a transverse wavelength $\Lambda$ will reproduce itself after the so-called Talbot distance, $z_{T}=2 \Lambda^{2} / \lambda$, where $\lambda$ is the light wavelength. At a quarter of this distance a phase modulation is transferred into an amplitude modulation 27 giving the feedback distance, $z_{T} / 4=2 d$, where optimal positive feedback occurs for a self-focusing medium (neglecting nonlinear absorption). This leads to a length scale of $\Lambda \propto \sqrt{4 d \lambda 25 \mid 26}$. This length scale selection can be also qualitatively understood in simple terms because the diffraction angle of a $\Lambda$ sized structure is $\approx \lambda / \Lambda$ and hence after a distance $2 d$ a transverse interval of $\Lambda \approx 2 d \lambda / \Lambda$ will be coupled by diffraction. If the thickness, $L$, of the medium is not negligible compared to the free space propagation distance, diffraction within the medium also plays a role. The qualitative reasoning given above remains valid, although $L$ affects the length scale of the pattern (see Sect. I.C of the Supplementary Material).

Probing the atomic density pattern. It was checked that the instability is not a polarization instability, i.e. the spontaneous sidebands have the same polarization as the pump beam. This enables us to introduce a polarizing beam splitter (PBS) in the feedback loop so that we can use an orthogonally polarized probe beam to directly visualize the atomic density self-structuring resulting from the optomechanical instability. The probe beam is sent through the same fiber as the pump, typically $10 \mu$ s after the pump has been switched off. After transmission through the cloud, it is reflected by the PBS located before the mirror, and sent to a CCD (probe image). The probe beam does not reach the mirror and it is short $(10 \mu \mathrm{s})$ and weak $(s \ll 1)$ and thus does not exert any feedback on the atoms. Because of the large on-resonance OD of the cloud, standard absorption imaging cannot be employed to measure the transverse density distribution of the atoms. Instead, we take advantage of the dispersive behaviour of the cloud by using a probe detuning of $-7 \Gamma$ where absorption is reduced. The plane imaged by the probe detection optics is the same as for the pump beam. The fact that the pump and probe images are complementary illustrates that dispersive imaging is dominating the absorption effect and the resulting pump and probe patterns are compatible with a honeycomb atomic density structure, since light concentrates in regions of high refractive index, which for the negative probe detuning corresponds to regions of high atomic density and for blue detuning to the voids (see Fig. 2 .

[1] Brennecke, F., Ritter, S., Donner, T. \& Esslinger, T. Cavity Optomechanics with a Bose-Einstein Condensate. Science 322, 235-238 (2008).

[2] Favero, I. \& Karrai, K. Optomechanics of deformable optical cavities. Nature Phot. 3, 201-205 (2009).

[3] Ritsch, H., Domokos, P., Brennecke, F. \& Esslinger, T. Cold atoms in cavity-generated dynamical optical potentials. Rev. Mod. Phys. 85, 553-601 (2013).

[4] Domokos, P. \& Ritsch, H. Collective cooling and selforganization of atoms in a cavity. Phys. Rev. Lett. 89, 253003 (2002).

[5] Black, A. T., Chan, H. W. \& Vuletic, V. Observation of collective friction forces due to spatial self-organization of atoms: from Rayleigh to Bragg scattering. Phys. Rev. Lett. 91, 203001 (2003).

[6] von Cube, C. et al. Self-synchronization and dissipationinduced threshold in collective atomic recoil lasing. Phys. Rev. Lett. 93, 083601 (2004).

[7] Baumann, K., Guerlin, C., Brennecke, F. \& Esslinger, T. Dicke quantum phase transition with a superfluid gas in an optical cavity. Nature 464, 1301-1307 (2010).

[8] Inouye, S. et al. Superradiant Rayleigh Scattering from a Bose-Einstein Condensate. Science 285, 571-574 (1999).

[9] Greenberg, J. A., Schmittberger, B. L. \& Gauthier, D. Bunching-induced optical nonlinearity and instability in cold atoms. Opt. Exp. 19, 22535 (2011).

[10] Gopalakrishnan, S., Lev, B. L. \& Goldbart, P. M. Emergent crystallinity and frustration with Bose-Einstein condensates in multimode cavities. Nature Phys. 5, 845-850 (2009).

[11] Gopalakrishnan, S., Lev, B. L. \& Goldbart, P. M. Atomlight crystallization of Bose-Einstein condensates in multimode cavities: Nonequilibrium classical and quantum phase transitions, emergent lattices, supersolidity, and frustration. Phys. Rev. A 82, 043612 (2010).

[12] Saffman, M. Self-induced dipole force and filamentation instability of a matter wave. Phys. Rev. Lett. 81, 65-68 (1998).

[13] Muradyan, G. A., Wang, Y., Williams, W. \& Saffman, M. Absolute instability and pattern formation in cold atomic vapors (2005). Nonlinear Guided Waves, OSA topical meeting technical digest, paper ThB29.

[14] Tesio, E., Robb, G. R. M., Ackemann, T., Firth, W. J. \& Oppo, G.-L. Spontaneous optomechanical pattern formation in cold atoms. Phys. Rev. A 86, 031801(R) (2012).

[15] Mendonça, J. T. \& Kaiser, R. Photon bubbles in ultracold matter. Phys. Rev. Lett. 108, 033001 (2012).

[16] Verkerk, P. et al. Designing optical lattices: an investi- 
gation with cesium atoms. Europhys. Lett. 26, 171-176 (1994).

[17] Brazowskii, S. A. Phase transition of an isotropic system to an nonuniform state. Sov. Phys. JETP 41, 85-89 (1975).

[18] Grynberg, G. Mirrorless four-wave mixing oscillation in atomic vapors. Opt. Commun. 66, 321-324 (1988).

[19] Ackemann, T. \& Lange, W. Non- and nearly hexagonal patterns in sodium vapor generated by single-mirror feedback. Phys. Rev. A 50, R4468-R4471 (1994).

[20] Dawes, A. M. C., Illing, L., Clark, S. M. \& Gauthier, D. J. All-optical switching in rubidium vapor. Science 308, 672-674 (2005).

[21] Smith, P. W., Ashkin, A. \& Tomlinson, W. Four-wave mixing in an artificial Kerr medium. Opt. Lett. 6, 284286 (1981).

[22] Reece, P. J., Wright, E. M. \& Dholakia, K. Experimental observation of modulation instability and optical spatial soliton arrays in soft condensed matter. Phys. Rev. Lett. 98, 203902 (2007).

[23] Man, W. et al. Optical Nonlinearities and Enhanced Light Transmission in Soft-Matter Systems with Tunable Polarizabilities. Phys. Rev. Lett. 111, 218302 (2013).

[24] Gupta, S., Moore, K. L., Murch, K. W. \& StamperKurn, D. M. Cavity nonlinear optics at low photon numbers from collective atomic motion. Phys. Rev. Lett. 99, 213601 (2007)

[25] Firth, W. J. Spatial instabilities in a Kerr medium with single feedback mirror. J. Mod. Opt. 37, 151-153 (1990).

[26] D'Alessandro, G. \& Firth, W. J. Spontaneous hexagon formation in a nonlinear optical medium with feedback mirror. Phys. Rev. Lett. 66, 2597-2600 (1991).

[27] Ciaramella, E., Tamburrini, M. \& Santamato, E. Talbot assisted hexagonal beam patterning in a thin liquid crystal film with a single feedback mirror at negative distance. Appl. Phys. Lett. 63, 1604-1606 (1993).

[28] Tesio, E., Robb, G. R. M., Ackemann, T., Firth, W. J. \& Oppo, G.-L. Kinetic theory for transverse optomechanical instabilities. Phys. Rev. Lett. (2014). In press.

[29] Ruiz-Rivas, J., Navarrete-Benlloch, C., Patera, G., Roldán, E. \& de Valcárcel, G. J. Dissipative structures in optomechanical cavities. arXiv:1212.1364v1 [physics.optics] 6 Dec 2012 (2012).

[30] Tesio, E., Robb, G. R. M., Ackemann, T., Firth, W. J. \& Oppo, G.-L. Dissipative solitons in the coupled dynamics of light and cold atoms. Opt. Exp. 21, 26144 (2013).

[31] Labeyrie, G. \& Bortolozzo, U. Light self-trapping in a large cloud of cold atoms. Opt. Lett. 36, 2158-2160 (2011).

\section{Acknowledgements}

The Strathclyde group is grateful for support by the Leverhulme Trust and EPSRC, the collaboration between the two groups is supported by the Royal Society (London). The Sophia Antipolis group acknowledges support from CNRS, UNS, and Région PACA.

\section{Author contributions}

GL lead the experimental effort and performed the experiment with the help of PG. They were joined by TA, AA, RK in data analysis. ET, GR, GLO and WJF performed the theoretical and computational analysis. TA conceived the experiment and coordinated the joint efforts with RK. All authors contributed to the discussion and interpretation of results and commented on the manuscript.

\section{Additional information}

The authors declare no competing financial interests. Supplementary information accompanies this paper at www.nature.com/naturephotonics. Reprints and permission information is available online at http://www.nature.com/reprints. Correspondence and requests for materials should be addressed to G.L. (guillaume.labeyrie@inln.cnrs.fr) or T.A. (thorsten.ackemann@strath.ac.uk). 


\title{
Optomechanical self-structuring in cold atomic gases - Supplementary material
}

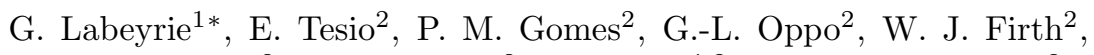 \\ G. R. M. Robb ${ }^{2}$, A. S. Arnold 2 , R. Kaiser ${ }^{1,2 *}$, and T. Ackemann ${ }^{2 *}$ \\ ${ }^{1}$ Institut Non Linéaire de Nice, UMR 7335 CNRS, \\ 1361 route des Lucioles, 06560 Valbonne, France and \\ ${ }^{2}$ SUPA and Department of Physics, University of Strathclyde, Glasgow G4 ONG, Scotland, UK
}

(Dated: V13 January 28, 2014)

\section{EXPERIMENTAL DETAILS AND ANALYSIS}

\section{A. Density pattern vs internal state pattern}

The instability described in this Letter relies on the optomechanical coupling between the light field and the atoms, whose response is well reproduced by a simple 2-level model. However, the complex internal structure of the atoms may also contribute additional nonlinearities. For instance, optical pumping between Zeeman substates is at the heart of the polarization instability observed in Refs 1]4. Hyperfine pumping has also been shown to play a role in self-lensing 5 . We observe in Fig. 2 patterns in the transmitted probe beam which we interpret as a direct consequence of spatial self-structuring of the cloud density distribution. To support this claim, we carefully checked alternative origins for the observed pattern, namely transverse inhomogeneous populations of long-lived internal states (Zeeman or hyperfine).

First, a $\mathrm{J} \rightarrow \mathrm{J}+1$ transition as investigated here does not possess dark states, which decouple from the light-atom interaction and are responsible for polarization preserving Zeeman patterns $\frac{677}{}$ and polarization instabilities 1 [1] 47 described before in hot $\mathrm{Na}$ and $\mathrm{Rb}$ vapour in the vicinity of the $\mathrm{D}_{1}$-line. Hence Zeeman pumping serving as the basis of a nonlinear effect is not expected. Second, the formation of high-contrast Zeeman-state gratings is expected to be highly polarization- and magnetic fielddependent since it requires the use of a carefully controlled polarization and the application of a magnetic field of appropriate orientation and strength. On the contrary, in our experiment the patterns are observed to be quite independent of the polarization configuration: we observed patterns for a lin // lin (same linear polarization for input and retro-reflected beams), $\sigma^{+}-\sigma^{+}$(same circular polarization) and $\sigma^{+}-\sigma^{-}$(orthogonal circular polarizations) configuration. For the lin // lin configuration, we observed no difference between patterns obtained with a well-compensated magnetic field and with an applied bias field of a few Gauss of various orientations (parallel to the pump propagation axis, parallel to the pump polarization, or orthogonal to both). In addition it was checked that the instability modes have the same polarization as the pump mode, i.e. there is no polariza-

\footnotetext{
*To whom correspondence should be addressed.
}

tion instability. These tests appear to rule out Zeeman induced population patterns.

We also checked the population of the lower $F=1$ hyperfine state after the pump pulse and found that it is rather small (at most 10\%) and cannot account for the large modulation observed in the probe transmission. A transmission image using light at the repumping transition frequency revealed no significant spatial structure in the $F=1$ population, ruling out hyperfine pumping as a mechanism for pattern formation.

Finally, the slow decay of the probe pattern with increasing pump-probe delay (see Fig. 3) rules out an occupation of the excited state as the origin of the probe modulation and is compatible with a wash-out of the density pattern due to the velocity distribution of the atoms.

\section{B. Optomechanical vs two-level nonlinearity}

As discussed in the article, the time scales for the growth of the probe pattern (Fig. 4) and its decay (Fig. 3a) indicate that there is an optomechanical instability leading to transverse spatial bunching. As discussed in more detail below (Sec. II), this instability can develop even in the absence of any intrinsic nonlinear optical effect relying on the population of the excited state ('two-level' or 'electronic' optical nonlinearity), in the linear optical regime. For pump intensities in the few $100 \mathrm{~mW} / \mathrm{cm}^{2}$ range, however, the two instability mechanisms (optomechanical and two-level) coexist and can then be distinguished via their different time scales by varying the pump pulse duration as shown in Fig. 4.

Further evidence is provided in Fig. S1, where we compare the $O D$ thresholds for short $(1 \mu \mathrm{s})$ and long $(200 \mu \mathrm{s})$ pump pulses. In this measurement, the $O D$ of the cloud is varied by tuning the time delay between the extinction of the MOT and the pump pulse. Because of the cloud's ballistic expansion after release from the MOT, the $O D$ monotonically decreases with increasing delay. The pump intensity is $487 \mathrm{~mW} / \mathrm{cm}^{2}$, and its detuning is $+6 \Gamma$. For the short pulse (two-level instability), the contrast of the pump pattern increases above a threshold $O D \approx 89$. In the case of the longer pulse, due to the optomechanical mechanism, the threshold $O D$ is considerably reduced to a value around 19 . 


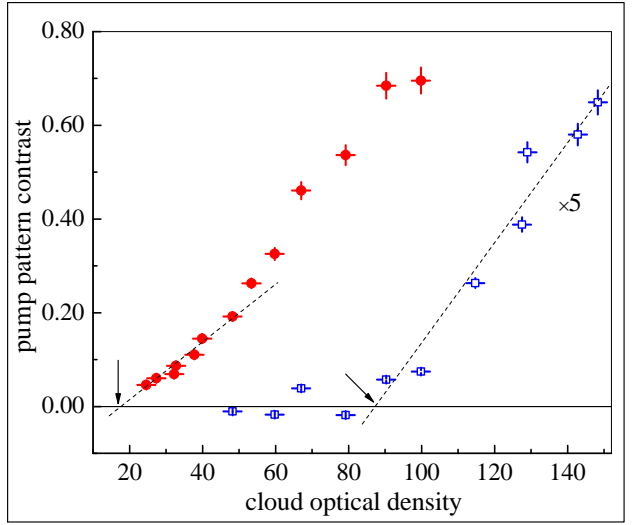

Fig S 1: Instability threshold behaviour versus cloud optical density. The measured evolution of the pattern contrast with $O D$ is compared for two pump pulses: a short pulse $(1 \mu \mathrm{s}$, blue squares), and a long one $(200 \mu \mathrm{s}$, red circles). In the latter case, the optomechanical mechanism strongly reduces the threshold $O D$. The pump parameters are: $\delta=+6 \Gamma$, $I=487 \mathrm{~mW} / \mathrm{cm}^{2}$.

\section{Possible influence of longitudinal bunching and thick medium}

For parallel incident and reflected pump polarizations, a standing wave establishes inside the cloud possibly leading to longitudinal spatial bunching of the atoms (see, e.g. 8 for counter-propagating pump beams at $\delta<0$ ). Qualitatively, however, longitudinal bunching seems to play a rather unimportant role in our experiment since similar patterns are observed for parallel polarizations (presence of a standing wave) and orthogonal polarizations (no standing wave in intensity, but in polarization state), either linear or circular.

A possible explanation is that atoms are accelerated in the longitudinal direction by the pump, which prevents their confinement in the longitudinal standing wave. Indeed, there is an intensity imbalance (typically 10-20\%) between the incident pump beam and the retro-reflected one, due to photon scattering by the cloud, which yields a large radiation pressure acting on the atoms in the longitudinal direction. This pushing effect has no impact in the transverse directions, where only regular heating due to pump photon scattering occurs. We also note that the blue-detuned longitudinal molasses created by the pump beam and retro-reflected beam does not provide cooling forces along the optical axis and hence does not favour confinement in the standing wave. The time scales for the growth of the probe pattern (Fig. 4) and its decay (Fig. 3a) indicate that transport processes take place on the transverse length scale of $100 \mu \mathrm{m}$ and not on wavelength scales $(0.4 \mu \mathrm{m})$, where one expects times in the sub-microsecond to $10 \mu$ s range. These hypotheses will be investigated in future work.

We develop in Sec. II the simplest theory which does not take into account a wavelength-scale density grating and and find good agreement with thresholds observed in the experiment, further supporting the idea that the principal mechanism is captured by the model. We mention that the same assumption for the density was made in the proposal for counterpropagating beams in $\underline{9}$.

Fig. 3b clearly establishes a dependence of the pattern length scale $\Lambda$ on the mirror distance $d$, but a careful analysis shows that it is only qualitatively described by a theory in which the medium is diffractively thin and all diffraction takes place in the vacuum feedback loop, since in the present experiment the mirror distance is comparable to the medium thickness $(\simeq 1 \mathrm{~cm})$. The effects of medium thickness are studied here by including mirror feedback in a model of counter-propagation instabilities in a Kerr slab 10 , i.e. a thick medium with $n=n_{0}+n_{2} I$ where the $n_{2}$ might originate from any nonlinearity (here optomechanical or two-level). This changes the boundary conditions on the forward and backward beams and hence modifies the growth condition for a non-trivial solution of the linearized equations for transverse perturbations of wavenumber $q$. This condition determines the threshold for instability of the homogeneous solution, and is typically an undulatory function of $q$. Its minima correspond to 'modes' $q_{\text {min }}$ with locally minimum threshold and in our model depend on the mirror distance $d$ as well as on the thickness and other parameters of the medium.

Since $d$ enters only through the differential phase shift (proportional to $q^{2} d$ ) between the on-axis pump beam and the $q$-sideband, the theory allows negative values of $d$. This can be achieved in the experiment by imaging the feedback mirror to a plane inside, or even beyond, the atom cloud. As shown in Fig. 3b, the dependence on $d$ for the lowest-wavenumber mode (squares) is in good agreement with our experimental data (circles) across a broad range of positive and negative mirror distances. In this figure the zero of the $d$-axis corresponds to the centre of the nonlinear medium. This good agreement confirms that the cause of the instability is the conversion of phase to intensity modulation in the feedback loop. The tunability of $\Lambda$ is a specific feature of the single mirror-feedback scheme, absent from the counterpropagating scheme with two input beams and no optical feedback ${ }^{8}[10]$.

\section{THEORETICAL MODEL AND NUMERICAL RESULTS}

We have undertaken a thorough theoretical investigation of the optomechanically-driven instability. Previous studies of optomechanical instabilities assumed strong velocity damping due to the presence of optical molasses $9 \sqrt{11}$. However, our experiment showed spontaneous symmetry breaking in the absence of such damping, with the MOT beams switched off during the interaction. We then describe the atomic dynamics in terms of a molassesfree, collision-less Boltzmann equation with a nonlinear term driven by the dipole force. The state of the 

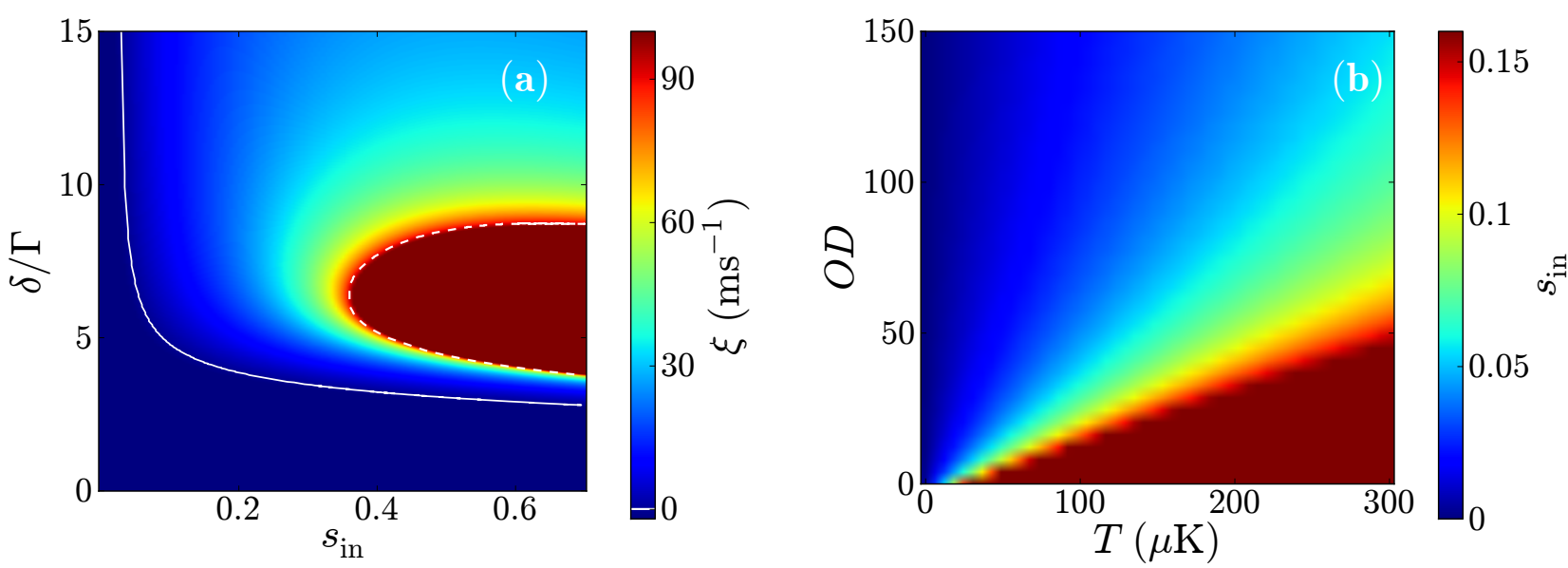

Fig S 2: Optomechanical instability: theoretical results. a) Instability phase diagram in a plane spanned by the detuning $\delta / \Gamma$ and the input saturation parameter $s_{\text {in }}$ (input intensity normalized to the saturation intensity at that detuning). The parameters are: $R=1, O D=150, T=290 \mu \mathrm{K}, d=5 \mathrm{~mm},|\mathbf{q}|=q_{\mathrm{c}}$. A 'fast' $(\xi \simeq \Gamma)$ internal-state instability is obtained inside the dashed white line. The instability threshold for self-structuring is displayed by the solid white line. Note that the dark blue coding (below the white line in the colour bar) denotes negative growth rates, i.e. the system is below threshold. b) Intensity threshold as a function of optical density and temperature for the same parameters as in a) and $\delta=6 \Gamma$. Lower temperatures lead to lower intensity and $O D$ thresholds for the instability.

cloud is described by its phase-space distribution function $f=f(\mathbf{x}, \mathbf{v}, t)$ (with $\mathbf{x}$ and $\mathbf{v}$ position and velocity vectors in the plane transverse to the field propagation, respectively), its dynamics being given by

$$
\frac{\partial f}{\partial t}+\mathbf{v} \cdot \frac{\partial f}{\partial \mathbf{x}}+\frac{\mathbf{F}_{\mathrm{dip}}}{M} \cdot \frac{\partial f}{\partial \mathbf{v}}=0 .
$$

Here $M$ is the atomic mass and $\mathbf{F}_{\text {dip }}=-\partial_{\mathbf{x}} U_{\text {dip }}$ the dipole force with $U_{\text {dip }}=(\hbar \delta / 2) \log (1+s(\mathbf{x}, t))$, and $s$ is the saturation parameter introduced in the main article and is given by the intensity scaled to the saturation intensity at that detuning. The spatial density $\rho(\mathbf{x}, t)$ is obtained by integrating $f$ over the entire velocity space, with the normalization chosen so that the spatially homogeneous solution corresponds to $\rho=1$. The saturation parameter $s$ is given by the sum of the suitably normalized intensities of the incident forward field $g_{F}$ and the backward field $g_{B}$, i.e. $s=\left|g_{F}\right|^{2}+\left|g_{B}\right|^{2}$ (neglecting longitudinal interference effects, see above). As $g_{B}$ depends on the density $\rho$ (see below), this closes the feedback loop. We stress that - via the normalization of $s$ - the normalization of $g_{F}$ and $g_{B}$ is also detuning dependent.

Neglecting diffraction effects inside the cloud (thin medium approximation), the interaction between a forward pump field of amplitude $g_{F}$ and the cloud of lasercooled two-level atoms is described by the following equation

$$
\frac{\partial g_{F}}{\partial z}=-\frac{O D(1-2 i \delta / \Gamma)}{2 L\left[1+4(\delta / \Gamma)^{2}\right]} \frac{\rho}{(1+s)} g_{F},
$$

where $L$ is the medium thickness and $O D$ the optical density. Eq. (2) describes absorption and dispersion in the atomic cloud. The $1 /(1+s)$ nonlinear term originates from adiabatic elimination of the internal-state dynamics, which is justified by the fact that the atomic motion occurs on a timescale much larger than $\Gamma^{-1}$ (see also Eq. (1) in the main article). To obtain $g_{B}$, we first integrate Eq. (2) under the assumption of a longitudinally homogeneous $\rho$ and $s$ and obtain the transmitted field $g_{T}$ at the exit face of the medium. The free-space propagation to the mirror (distance $d$ ) and back can be solved exactly in Fourier space, and the backward field before re-entrance in the medium is given by:

$$
g_{B}(q)=\sqrt{R} e^{i d q^{2} / k_{0}} g_{T}(q),
$$

where $R$ denotes the mirror reflectivity.

A stationary homogeneous solution for the system is determined by $s=s_{h}, f=f_{0}(\mathbf{v})$, where $f_{0}(\mathbf{v})$ is the initial velocity distribution of the cloud. An implicit expression for the homogeneous intensity, $s_{h}$, is obtained from Eqs. (2), (3) by setting $\rho=1$ and evaluated via a zero-finding routine. We introduce perturbations in the distribution function and the backward field as $f=f_{0}+f_{1}(\mathbf{x}, t), g_{B}=g_{B}^{(h)}\left[1+b_{1}(\mathbf{x}, t)\right]$, and linearize Eqs. (1), (2), (3) about the homogeneous solution. The dispersion relation $\omega=\omega(|\mathbf{q}|)$ (q being the transverse wavevector) and the threshold for the instability are then determined using linear stability analysis. The most unstable wavenumber (with largest growth rate) is only slightly shifted from the one obtained from a purely dispersive theory, $q_{\mathrm{c}}=\sqrt{\pi k_{0} / 2 d}$, see e.g. Ref. ${ }^{12}$. The corresponding spatial scale of the emerging patterns is in good qualitative agreement with the experimental results; a quantitative agreement is obtained by taking into 
account the thickness of the medium, see Fig. $3 \mathrm{~b}$ and Sect. IC

Fig. S2a shows the growth rate $\xi=\operatorname{Im}(\omega)$ for perturbations at the critical wavenumber $q_{c}$ and parameters close to our experiment using the normalized detuning $\delta / \Gamma$ and the saturation parameter $s_{\text {in }}$ of the incident pump beam (proportional to the intensity of the forward beam at the input facet of the cloud) as control parameters. The condition $\xi>0$ parametrizes unstable regions (upper right of the solid white line). There is no instability on resonance and the threshold drops monotonically with increasing detuning, reaching an asymptotic value at large $\delta / \Gamma$ (in units of the saturation parameter; in terms of the unscaled input intensity the threshold intensity increases asymptotically quadratically with detuning). Beyond threshold there is a significant region of pump power in which the growth rate is fairly low (below $100 \mathrm{~ms}^{-1}$ ). For an intermediate range of detunings, increasing the pump intensity, the growth rate suddenly increases dramatically in a small range of pump intensity before settling to a broad plateau (the dark brown region in Fig. S2a) with a peak value of about $\xi \sim 10^{4} \mathrm{~ms}^{-1} \sim O(\Gamma)$, taken from a purely two-level model at $O D=150$. (The growth rate in the approximation of Eq. (2) diverges as adiabatic elimination of the internal state dynamics essentially sets $\Gamma \rightarrow \infty$.) This region is essentially identical to the one (indicated by the white dashed line) in which the spatial instability can be triggered only by the two-level, internal-state nonlinearity (corresponding to $\rho=1$ ). Physically this corresponds to transverse structures emerging on the timescale of the atomic lifetime, encoded in the populations and coherences of the atoms. This is the situation of curves 1 and 2 of Fig. 4 of the article, in which the light pattern was already established after one microsecond, without the formation of a density pattern. In contrast, in between the solid and dashed white lines the growth rates are compatible with optomechanical time scales. This is the situation of curve 4 of Fig. 4 of the article, where light and density pattern develop together, i.e. the instabil- ity is optomechanically driven. Indeed, it turns out that the instability still exists if the two-level term $(1+s)$ in Eq. (2) is neglected. In addition, the thresholds for the purely optomechanical and the mixed case are quite similar (and very different from the purely two-level one) indicating that indeed the optomechanical nonlinearity is the main driver in this parameter range.

In Fig. S2b we show the dependence of the intensity threshold $s_{\text {in }}$ on the temperature and the optical density. As expected, lower temperatures result in lower intensity and $O D$ thresholds for the instability. It is remarkable to see that in spite of the approximations made, the theoretical model predicts the lowest threshold at around $s_{\text {in }}=0.04$ for parameters close to the experiment $(\delta=10 \Gamma, O D=150, T=300 \mu \mathrm{K})$, i.e. well within the same order of magnitude of that experimentally observed (curve $(4)$ in Fig. 4 corresponds to $s_{\text {in }} \approx 0.06$ ). For the parameters of Fig. S1, the predicted threshold in optical density is around 28 , i.e. also in good qualitative agreement with the experimental observation of about 19 .

The treatment presented here shows that, depending on detuning, above some critical saturation level internalstate nonlinearities might initiate the pattern formation and determine the timescale of the initial dynamics, but that close to threshold the instability is dominantly optomechanical in nature. In addition, we stress again that nonlinear density redistribution effects can lead to symmetry-breaking instabilities even in the absence of any internal-state nonlinearity (that is, in the low saturation regime). For large detuning it is also possible to neglect attenuation of the forward beam due to absorption, leading to an unitary operator in Eq. (2). This leads to an ultra-simplified and yet general model for self-structuring, which will be presented elsewhere ${ }^{13}$. Internal-state effects are important for intermediate to high values of the saturation parameter $s_{\text {in }}$ in regimes far beyond threshold, but lead to fast instabilities that can be separated from the basic self-structuring mechanism.
[1] Gauthier, D. J., Malcuit, M. S. \& Boyd, R. W., Polarization instabilities of counterpropagating laser beams in sodium vapor. Phys. Rev. Lett. 61, 1827-1830 (1988).

[2] Petrossian, A., Pinard, M., Maître, A., Courtois, J.-Y. \& Grynberg, G., Transverse pattern formation for counterpropagating laser beams in rubidium vapour. Europhys. Lett. 18, 689-695 (1992).

[3] Grynberg, G., Maître, A., \& Petrossian, A., Flowerlike patterns generated by a laser beam transmitted through a rubidium cell with a single feedback mirror. Phys. Rev. Lett. 72, 2379-2382 (1994).

[4] Aumann, A., Büthe, E., Logvin, Yu. A., Ackemann, T. \& Lange, W., Polarized patterns in sodium vapor with single mirror feedback. Phys. Rev. A 56, R1709-R1712 (1997).

[5] Labeyrie, G., Gattobigio, G.-L., Chanelière, T., Lippi,
G.-L., Ackemann, T. \& Kaiser, R., Nonlinear lensing mechanisms in a cloud of cold atoms. Eur. Phys. J. D 41, 337-348 (2007).

[6] Ackemann, T. \& Lange, W. Non- and nearly hexagonal patterns in sodium vapor generated by single-mirror feedback. Phys. Rev. A 50, R4468-R4471 (1994).

[7] Ackemann, T. \& Lange, W. Optical pattern formation in alkali metal vapors: Mechanisms, phenomena and use. Appl. Phys. B 72, 21-34 (2001).

[8] Greenberg, J. A., Schmittberger, B. L. \& Gauthier, D. Bunching-induced optical nonlinearity and instability in cold atoms. Opt. Exp. 19, 22535 (2011).

[9] Muradyan, G. A., Wang, Y., Williams, W. \& Saffman, M. Absolute instability and pattern formation in cold atomic vapors. Nonlinear Guided Waves, OSA topical meeting technical digest, paper ThB29 (2005). 
[10] Geddes, J. B., Indik, R., Moloney, J. V. \& Firth, W. J. Hexagons and squares in a passive nonlinear optical system. Phys. Rev. A 50, 3471-3485 (1994).

[11] Tesio, E., Robb, G. R. M., Ackemann, T., Firth, W. J. \& Oppo, G.-L. Spontaneous optomechanical pattern formation in cold atoms. Phys. Rev. A 86, 031801(R) (2012).
[12] Firth, W. J. Spatial instabilities in a Kerr medium with single feedback mirror. J. Mod. Opt. 37, 151-153 (1990).

[13] Tesio, E., Robb, G. R. M., Ackemann, T., Firth, W. J. \& Oppo, G.-L. Kinetic theory for transverse optomechanical instabilities. Phys. Rev. Lett. (2013). In Press. 